\title{
COMENTÁRIO EDITORIAL O TÍTULO, RESUMO E PALAVRAS-CHAVE DOS ARTIGOS
}

Fernando Antonio Ribeiro Serra Editor Científico RIAE

Universidade Nove de Julho - UNINOVE Programa de Pós-Graduação em Administração

\author{
Manuel Aníbal Silva Portugal Vasconcelos Ferreira \\ Editor Adjunto RIAE \\ Universidade Nove de Julho - UNINOVE \\ Programa de Pós-Graduação em Administração
}

Desde a edição v. 12 número 2, de agosto a setembro de 2013, da RIAE que temos publicado comentários editoriais. Estes comentários têm por objetivo fundamental passar aos nossos autores, e outros pesquisadores, especialmente os estudantes em programas de stricto sensu em Administração, sugestões e recomendações a seguir na elaboração dos seus artigos. A nossa expectativa é, assim, a de transmitir alguns ensinamentos, sempre que possível adaptados à realidade nacional, para melhorar a qualidade dos trabalhos desenvolvidos aumentando, assim, a chance de serem aceitos para publicação em bons periódicos nacionais e internacionais. A elaboração destes comentários tem nos proporcionado um grande aprendizado, pois não se trata de simplesmente passar a experiência acumulada ao longo de nossa carreira com a elaboração de projetos de pesquisa, elaboração de artigos e sua submissão a periódicos. Também tem originado novos projetos de pesquisa para entender, por exemplo, quais os motivos porque os artigos são rejeitados - ou seja, as lacunas mais frequentes que conduzem à rejeição - e as porque os pesquisadores buscam coautorias. Ainda, tem proporcionado o aprendizado que resulta quando buscamos entender o que outros pesquisadores internacionais analisam sobre estes temas.

Tem ficado claro nos eventos internacionais como o congresso da Academy of Management ou da Academy of International Business e nacionais, como o encontro anual da ANPAD - o ENANPAD, o interesse nestas temáticas. Evidência deste interesse é a grande assistência aos workshops sobre melhoria de artigos e encontro com editores. Pelo menos em parte podemos identificar algumas motivações para o interesse crescente. Por exemplo, a maior exigência dos Programas de stricto para que os professores pesquisem e publiquem em melhores periódicos. A maior sofisticação e exigência nos próprios periódicos 
nacionais classificados nos estratos Qualis mais altos (A2 e B1). Como autores, temos experimentado que as revisões pelos pareceristas demandam maior cuidado em todas as seções dos artigos. Ainda, a cada vez mais notória observação que a mobilidade inter-IES dos pesquisadores depende, pelo menos em parte, do seu histórico de publicações.

Neste comentário editorial vamos ao encontro de, e complementamos, aspectos que já foram aflorados em comentários editoriais anteriores ${ }^{\mathrm{i}}$. Especificamente, neste comentário editorial prestamos especial atenção a um aspecto que ainda continua a ser muito descurado que é o título, resumo e palavraschave dos artigos. Salientamos que esta importância tem emergido em diversos momentos nos pareceres dos artigos submetidos à RIAE. Estes componentes são cruciais para posicionar o leitor e para comunicar sua ideia de forma clara e eficiente (King \& Lepak, 2011). O foco deste comentário editorial é, assim, explorar alguns cuidados específicos na construção e redação do títulos, resumo e palavras-chave $\mathrm{e}^{\mathrm{ii}}$ dos artigos acadêmicos submetidos não apenas à RIAE, mas a todos os periódicos da área de Administração.

\section{ELEMENTOS DO PRIMEIRO CONTATO COM O LEITOR}

$\mathrm{Na}$ estrutura básica fundamental de um artigo acadêmico em Administração, o conjunto título, resumo e palavras-chave compõe o primeiro contato do leitor com o artigo. Quando o pesquisador busca pela bibliografia relevante usa bases de dados como a Proquest, Ebsco ou Scielo, qualquer outra como o Google Scholar. Nestas, insere um conjunto de palavras chave para definir a sua busca e o título do artigo é o primeiro elemento textual que utiliza para avaliar se o artigo pode ser relevante para o seu propósito. Só depois avança para a leitura do resumo. Efetivamente, algumas das bases de dados utilizam-se do título, resumo e palavras-chave como sendo os campos em que a busca é realizada. Sendo os primeiros elementos, são aqueles que despertam o interesse e provocam a decisão de continuidade de leitura para leitores. Estes elementos, é que são o primeiro passo para que um leitor ao se interessar pela leitura do restante do artigo, se este tiver conteúdo e qualidade, possa vir a referenciá-lo em seus trabalhos de pesquisa.

Apesar de serem elementos essenciais, o título, o resumo e as palavras-chave, em muitos casos, e talvez mais comumente entre autores menos experientes, são negligenciados e/ou desconectados em relação aos demais elementos do artigo. Ou seja, o título não é um bom reflexo do conteúdo efetivo do artigo. Ou, o resumo não é um reflexo coerente e sintético do que o artigo trata, não apresentando os elementos fundamentais. Outras vezes a deficiência é nas palavras-chave. A assunção é que o autor irá escolher um conjunto de palavras-chave (usualmente entre 4 e 6) que melhor distinguem o seu artigo. Estas palavras chave são usadas pelos motores de busca e para fins de indexação em sistemas bibliográficos.

\section{QUAL TÍTULO PARA O ARTIGO}

Uma das dúvidas usuais é que título dar ao artigo? Será melhor buscar um título atrativo, privilegiando a estética, ou um título informativo, que saliente o conteúdo? Pelo que foi exposto anteriormente, já ficou claro que é importante que o título capte a atenção do leitor. Assim, num primeiro momento, o impulso é de escrever um título empolgante e criativo. No entanto, mesmo nestes casos, é fundamental que o título "criativo" revele qual o conteúdo do artigo. De salientar, por exemplo, que mesmo em muitos formulários de revisão de artigos que são enviados aos pareceristas dos periódicos, uma das questões colocada é a adequação da ligação do título ao conteúdo do artigo submetido a avaliação. Assim, antes de o parecerista comentar aspectos específicos a cada parte do artigo, necessita responder a questão: "O título, o resumo/abstract e as palavras chave representam uma boa ideia do artigo como um todo?"

Como foi apontado no primeiro comentário editorial da RIAE (Ferreira, 2013: 4-5):

\begin{abstract}
"Ainda assim, alguns autores sacrificam a coerência entre título e conteúdo, o que pode ser uma falha letal no processo de revisão. Usando a analogia de um filme, quando vamos ao cinema usamos alguns indicadores para saber se o filme nos interessa, como: pelo título se estamos perante um filme de ação, de ficção, de suspense,_..., pelos atores reformamos essa percepção. Uma lógica semelhante é seguida nos artigos. Ao ler o título, o leitor, e o revisor na primeira instância, fica imediatamente enviesado para um determinado assunto, teoria ou tema e dificilmente entenderá um artigo cujo corpo não cumpra as expectativas expressas logo no título.
\end{abstract}

\section{Aspectos qualitativos do título}

Os autores internacionais que usualmente referenciamos sabem tornar um título atraente, pois procuram contar a história de seu artigo logo no título ou, pelo menos, dar uma boa indicação de qual será essa história. Ou seja, no título já se incluem elementos que fazem a ligação com o que está sendo estudado, passando uma ideia ao leitor, ou revisor, do que poderá encontrar no corpo do artigo. Citamos como exemplo o trabalho que foi eleito como melhor artigo, em 2012, pelo Academy of Management Journal (AMJ), de Smetz, Morris e Grenwood (2012), "From practice to field: A multilevel model of practice-driven institutional change". O artigo no título remete para 
teoria institucional, indicando que propõe um modelo de mudança institucional a partir da prática. Outro exemplo é o artigo mais acessado da Revista de Administração de Empresas - RAE, de Renata Brito e Luiz Brito (2012), "Vantagem competitiva, criação de valor e seus efeitos sobre o desempenho". A leitura do título permite inferir imediatamente que o artigo trata de avaliar o efeito da vantagem competitiva e criação de valor sobre métricas de desempenho das empresas.

Portanto, o título é uma parte importante do artigo. Talvez uma das mais importantes, na realidade, porque precisa chamar atenção dos leitores potenciais nos poucos instantes em que passa os olhos. Sendo assim, podemos, agora, avançar com a sugestão que o título deve ser razoavelmente curto (Lebrun, 2004). Os exemplos selecionados anteriormente podem ser considerados títulos curtos, tendo cerca de 10 palavras (Russey, Ebel \& Bliefert, 2006). Porém a busca por colocar o título curto não pode sacrificar a sua clareza ou o seu poder de sinalizar conteúdo. No exemplo anterior do artigo publicado no AMJ, de Smetz et al. (2012), apesar de ter um título em inglês (idioma que chega a economizar $20 \%$ das palavras em relação ao português), o título contém 11 palavras.

Apesar de ser uma prática comum, é recomendável evitar abreviaturas no título (Hesson, 2013). Por exemplo, a abreviatura RSE pode ser colocada por extenso - Responsabilidade Social Empresarial.

É também recomendado colocar a informação nova no início do título. Por exemplo, no exemplo anterior do artigo publicado na RAC, a novidade vem antes dos “:” que separam o título (poderia também ser separado por um “-“, por exemplo), do restante das informações que descrevem e clarificam melhor o que se espera encontrar no artigo.

Vale apresentar a diferença entre Títulos Informativos e Títulos Explicativos. Títulos informativos têm como objetivo apresentar o propósito do estudo. Já os títulos explicativos objetivam apresentar os resultados dos estudos (Goldman, 2000). $\mathrm{O}$ exemplo do artigo publicado na era, por Brito e Brito, que apresentamos anteriormente tem mais característica de título informativo, que são os mais comuns nos estudos de administração, ao contrário dos predominantes nos estudos da área de saúde, por exemplo (Hesson, 2013). Um exemplo de título explicativo é o do artigo de Shen, Tang e Chen (2014), publicado no SMJ, "When the role fits: How firm status differentials affect corporate takeovers", pois remete ao resultado esperado do estudo.

\section{Tornando os títulos atrativos}

Reiteramos que mais que ser atrativo o título deve permitir posicionar o artigo e atrair leitores que tenham interesse no tema.

Já há algum tempo nos dedicamos a compreender melhor os fatores que influenciam a baixa produção internacional de autores brasileiros, com o intuito de também progredirmos com nossos trabalhos. Sobre este tema escrevemos, em 2008, um artigo cujo título foi bastante comentado por ser tanto atrativo como revelar o que trata. Neste artigo, da coautoria de Fernando Serra, Gabriela Fiates e Manuel P. Ferreira, publicado na Revista de Administração Mackenzie, o título "Publicar é difícil ou faltam competências? $O$ desafio de pesquisar e publicar em revistas científicas na visão de editores e revisores internacionais" revelava que tratávamos da dificuldade de publicar em bons periódicos internacionais.

Como se pode ver nos exemplos de títulos já inclusos neste comentário editorial, os títulos não usam verbos, são fragmentos gramaticais (Hesson, 2013). Na realidade há pequenos "truques" que pode usar para melhorar a sua atratividade. Por exemplo, o uso de gerúndio, ou infinitivo, nos artigos pode ajudar a criar a sensação de atividade e movimento. Por exemplo, um artigo recentemente publicado no Strategic Management Journal, de de Henisz, Dorobantu e Nartey (2014), a palavras "spining" ilustra esta situação: "Spinning gold: The financial returns to stakeholder engagement".

Por fim, sugerimos evitar mensagens que usem expressões culturalmente regionais, como trocadilhos e gírias. Embora estas expressões possam ser evidentes para os autores podem trazer dificuldade para os restantes leitores. Lembre-se que mesmo tentar traduzir uma palavra pode resultar em algo bem estranho. Evite, também, adjetivar o título escrevendo palavras que apontem que algo é "novo", ou "melhorado", pois qualquer trabalho de pesquisa precisa melhorar algo ou trazer algo de novo. Esta novidade é, pelo menos em parte, a contribuição. Sugerimos, também, evitar palavras fortes demais como "solução", “cura", etc. (Hesson, 2013).

\section{PREPARAR O RESUMO}

O resumo é uma das partes mais lidas, logo a seguir ao título. Ao ler o resumo, um potencial leitor espera encontrar uma síntese dos principais aspectos do artigo. Assim, o resumo também precisa ter algum elemento de atratividade mas, fundamentalmente, de informativo sobre o que o artigo trata, como trata, o que faz metodologicamente, o que conclui e qual a sua contribuição.

Então, como construir um resumo? Importa começar por deixar claro que não existe uma regra universal para como escrever um resumo. No entanto, há normas do que se espera esteja contemplado no resumo. Em essência o próprio título já diz que é um "resumo". No entanto, não é objetivamente possível fazer um resumo de todo o artigo em cerca de seis frases. Assim, a nossa sugestão é que o resumo siga a estrutura tradicional de um artigo, inclusive, se possível indicando qual a fundamentação teórica. O que isto significa é que $\mathrm{O}$ resumo deve ter uma indicação dos seguintes elementos: questão de pesquisa método 
utilizado, principais resultados, discussão dos resultados voltada para as implicações para teoria e prática (ou seja, a contribuição).

\section{Os elementos do resumo}

Como nos títulos, os resumos dos artigos científicos devem ser informativos. Assim, devem proporcionar uma perspectiva global de cada uma das seções do artigo, como mencionámos, de forma sintética e clara. A seguir apresentamos uma lista dos principais elementos a considerar quando redige o resumo:

- Objetivo: apresentar os aspectos fundamentais da pesquisa, por um texto, pela questão de pesquisa;

- Método: descrever suscintamente o método, não deixar de mencionar, no caso de artigos quantitativos, a amostra, os procedimentos de coleta, os procedimentos de análise dos dados, as variáveis, os instrumentos usados ou as fontes dos dados.

- Resultados: apontar (eventualmente descrevendo) os principais resultados da pesquisa;

- Discussão: apresentar as conclusões, as limitações e implicações do estudo.

Os resumos podem ser estruturados e não estruturados. Esta não é tanto uma questão de opção do pesquisador, mas mais das normas definidas pelo periódico. Alguns periódicos têm criado um formato próprio para apresentação de resumos estruturados. No entanto, uma estrutura muito rígida pode ter impacto negativo na comunicação de artigos mais qualitativos e conceituais (Hesson, 2013).

A seguir apresentamos dois exemplos de resumos, estruturado e não estruturado. No artigo "Organizational decline: A yet largely neglected topic in organizational studies", de Serra, Ferreira e Almeida (2013), publicado na Management Research, o resumo apresentado é estruturado seguindo a norma dos periódicos da editora Emerald. Note-se que um resumo estruturado permite ao leitor identificar rapidamente cada um dos aspectos centrais. Vale ressaltar que se trata de um resumo grande, com mais de 300 palavras.

Objetivo - Neste artigo revemos a literatura em declínio organizacional e aspectos relacionados (turnaround, insolvência, etc.) e analisamos a influência teórica na investigação acadêmica. Examinamos a contribuição teórica e os autores mais influentes para compreender melhor a estrutura intelectual que liga teorias e autores que pesquisaram em declínio organizacional. Este estudo permite não apenas compreender o estado da arte da investigação, mas também, com base nas lacunas identificadas, descobrir e orientar estudos futuros em declínio organizacional.
Design/Metodologia - Metodologicamente realizamos um estudo bibliométrico envolvendo análises de citações e cocitações para identificar interligações entre autores e teorias utilizadas. O estudo bibliométrico incidiu sobre 18 revistas de gestão com mais alta reputação. A nossa amostra de104 artigos foi recolhida na ISI Web of Knowledge.

Resultados - Identificamos quatro clusters de temas investigados e concluímos que as teorias evolucionárias suportam um número considerável de trabalhos, incluindo estudos sobre os efeitos da inércia, isomorfismo e adaptação. As perspectivas sociológicas também têm sido usadas, nomeadamente no estudo do ajustamento das estruturas internas e ações para melhorar o posicionamento. Outras investigações são baseadas em trabalhos de aprendizagem e tomada de decisão, além de estratégias de turnaround para evitar, ou sair, do declínio.

Limitações/implicações - As principais limitações referem-se ao método e amostra. Embora utilizemos 18 revistas de gestão de elevada reputação, estas não incluem todo o conhecimento produzido.

Originalidade/valor - Mesmo as melhores e mais reputadas empresas, não apenas as pequenas empresas empreendedoras, enfrentam declínio e eventual extinção. Apesar de o declínio organizacional ser uma realidade observável, há uma notável escassez de investigação sobre o tema. O declínio organizacional é mais frequentemente estudado em pequenas empresas e atribuído às desvantagens de juventude (liability of newness) e a carências de diversos tipos de recursos físicos, tecnológicos, financeiros e sociais. A expectativa de identificarmos trabalhos usando a Visão Baseada nos Recursos sobre o declínio não se verificou, talvez porque desde os anos de 1990 diminuiu o interesse dos investigadores em estratégia de estudar declínio. Ainda assim, investigadores em ecologia populacional continuam a ter as questões do declínio no centro do seu trabalho.

$\mathrm{O}$ artigo mais acessado do SMJ é o "Dynamic capabilities and strategic management", de Teece, Pisano e Shuen (1997). Este artigo, seguindo as normas do SMJ tem um resumo não estruturado

The dynamic capabilities framework analyzes the sources and methods of wealth creation and capture by private enterprise firms operating in environments of rapid technological change. The competitive advantage of firms is seen as resting on distinctive processes (ways of coordinating and combining), shaped by the firm's (specific) asset positions (such as the firm's portfolio of difficult-to-trade knowledge assets and complementary assets), and the evolution path(s) it has adopted or inherited. The importance of path dependencies is amplified where conditions of increasing returns exist. Whether and how a firm's competitive advantage is eroded depends on the stability of market demand, and the ease of replicability (expanding internally) and imitatability (replication by 
competitors). If correct, the framework suggests that private wealth creation in regimes of rapid technological change depends in large measure on honing internal technological, organizational, and managerial processes inside the firm. In short, identifying new opportunities and organizing effectively and efficiently to embrace them are generally more fundamental to private wealth creation than is strategizing, if by strategizing one means engaging in business conduct that keeps competitors off balance, raises rival's costs, and excludes new entrants.

\section{Alguns cuidados na redação do resumo}

Redigir um bom resumo é um desafio. Não só é difícil resumir um artigo no seu essencial em poucas frases, demonstrando e salientando a sua relevância, mas, também, pelos aspectos relacionados ao tamanho e qualidade do texto escrito.

O desafio de dimensão fica claro pelas instruções apresentadas em geral nas instruções dos periódicos. Um resumo é breve por natureza. Os periódicos acadêmicos, em geral, delimitam a extensão do resumo a cerca de 250 palavras. Desta forma, considerando a estrutura tradicional, contendo apresentação de questão de pesquisa, método, resultados e discussão, sugerimos que comece por tentar escrever cerca de uma frase por parte. Depois, na revisão, melhore as frases. Nesta recomendação, vale mencionar que se precisar estender e houver oportunidade pelas instruções de publicações do periódico, dar maior atenção aos elementos de discussão (implicações para teoria e prática, originalidade e limitações do trabalho- muitas vezes as limitações podem ser aspectos contextuais que delimitam a teoria).

Ainda que seja implícito em nossas sugestões, é preciso clarificar que deve evitar o uso de abreviações, acrônimos, citações e referências, mas, também, tabelas ou figuras e fórmulas. Note que mesmo acrônimos comuns e genericamente conhecidos pela comunidade - como RBV, para Resource-Based View - devem ser escritos por extenso na primeira vez que surgem no texto.

O resumo também não é um campo em que se deve expor em detalhe a revisão de literatura. Talvez mais especialmente os estudantes, por vezes, alongamse a apresentar a revisão de literatura no resumo. Além de ocupar espaço, o excesso de teoria no resumo pode distanciar o resumo do seu papel informativo. O que é adequado é apontar qual o posicionamento conceitual do artigo, não sendo (usualmente) necessário entrar em maiores detalhes que serão explanados em outra seção.

O resumo também não necessita de demasiada explicação de qual a importância da pesquisa no tema. Este elemento explicativo irá surgir na introdução.

Swales e Feate (2004) argumentam que os resumos devem usar verbos no presente e presente perfeito, mas em Ciências Sociais, também no passado.
Usando os exemplos dos resumos apresentados anteriormente utilizam no texto os verbos como: "revemos"; "analisamos", "examinamos", "analyses"; "is seen".

Vale relembrar que as palavras-chave, elemento explorado a seguir, devem ser usadas no resumo, com o intuito de manter o foco do artigo e aumentar a probabilidade de o artigo ser encontrado pelos motores de busca que usamos na pesquisa bibliográfica.

\section{DEFININDO AS PALAVRAS-CHAVE}

Já salientámos a importância de uma boa escolha das palavras-chave para sinalizar o conteúdo do artigo a potenciais leitores. As palavras-chave também são usadas pelos motores como indexadores, sendo um elemento fundamental quando fazemos a busca de bibliografia. Pela nossa experiência como editores e pareceristas ad hoc para dezenas de periódicos nacionais e internacionais, constatamos que os autores se preocupam pouco em examinar as melhores opções de palavras-chave para seus artigos. Pela importância que podem ter no impacto conjunto com o título e o resumo, acreditamos que merecem maior atenção.

Russel (2004) sugeriu algumas formas para selecionar as palavras-chave. Especificamente, sugeriu que estejam focadas nos tópicos mais importantes do artigo (podem, inclusive, ser destacadas do título e do resumo, se tiverem sido bem elaborados). Russel também sugeriu observar outros bons trabalhos publicados sobre tema similar e observar quais as palavras-chave que usaram. Ao fazer este exercício de "imitação" o que realmente pretende é observar quais as palavras que outros autores consideraram melhor refletir o artigo. Este exercício também ajudará a identificar quais as palavras que ajudam a posicionar o artigo numa dada linha de pesquisa. Vale ressaltar que as palavras-chave podem estar presentes no título ou no texto do resumo. Nesta busca por palavras-chave mais adequadas, podem existir outras palavras com significado similar que apareçam com frequência. Neste caso, se pode utilizar uma no título e outra no resumo, de forma a aumentar a chance de busca.

\section{Classificação das palavras-chave}

As palavras-chave podem ser classificadas em gerais, intermediárias ou específicas (Lebrun, 2007). Se pensarmos nos pesquisadores da nossa comunidade específica - provavelmente mais conhecedora do assunto que tratamos no artigo - as palavras-chave específicas seriam as mais importantes, pois deixam bem claro o tópico que está sendo abordado. Ou seja, as palavras-chave para estes pesquisadores que estudam aspectos ou fenômenos similares, ou com a mesma teoria, podem evidenciar a originalidade do trabalho. Para a restante comunidade pode ser necessário ter palavras-chave mais amplas que 
evidenciem, por exemplo, o contexto, a metodologia, ou a teoria. As palavras-chave intermediárias, também são importantes para pesquisadores da área, pois estes podem não conhecer o assunto específico, mas estarem interessados no tema.

As palavras-chave gerais podem parecer inadequadas, por serem muito gerais, mas são importantes pois ajudam os leitores que buscam o tema e são de outras áreas. Também podem ser especialmente úteis para novos pesquisadores que ainda não sabem bem o que buscar.

Nosso artigo "Estratégias de internacionalização sobre pressões institucionais: Um modelo conceitual sobre as imposições duais para legitimidade e conformidade", aprovado para publicação na RAC, pode servir de exemplo para verificar a diferença entre os tipos de palavras-chave colocadas em conjunto. $\mathrm{O}$ artigo tem apenas as quatro palavras-chave seguintes: Modos de entrada; Estratégia de internacionalização; Adaptação; Ambiente institucional. Não estão colocadas em ordem alfabética, pois optamos por coloca-las em ordem de prioridade, começando pela palavras-chave específica e que trata do tema fundamental do artigo: modos de entrada. A seguir, segundo nossa opção, colocamos a palavrachave mais genérica, que posiciona duplamente nossa área de estudo estratégia, e um dos temas líderes de nossa pesquisa, os negócios internacionais, pela composição "estratégia de internacionalização. Finalmente, com as duas demais palavras, que neste caso consideramos como intermediárias, compomos outro aspecto importante do artigo que se refere à influência do ambiente institucional nos modos de entrada, com a palavra "adaptação" e com "ambiente institucional". Este é um artigo conceitual fortemente baseado na análise do impacto do ambiente institucional sobre os modos de entrada das empresas, mostrando uma das facetas cruciais que é a forma como as empresas se adaptam a diversos estados do ambiente institucional nos países externos e em função das próprias pressões internas às empresas.

Outra sugestão é evitar palavras-chave grandes e confusas. Ao comprimir palavras-chave em nomes compostos, por exemplo, pode comprometer a clareza (lembrando que estará aparecendo nos demais elementos, título e resumo). Novamente, lembramos que gírias e jargões, restritos à cultura, são complicados para uso nas palavras-chave, como nos demais elementos abordados neste comentário editorial.

Em suma, como a maioria dos periódicos permite usar entre 3 a 6 palavras-chave, sugerimos optar por um mix de gerais, intermediárias e específicas. De salientar, no entanto, que se todas as palavras-chave escolhidas forem muito gerais, os experts da área podem não se interessar. Se forem muito específicas, os que não são experts podem nem sequer encontrar o artigo nas suas pesquisas bibliográficas (Lebrun, 2007).

\section{COMENTÁRIOS FINAIS}

A parte a discussão do produtivismo acadêmico, o nosso trabalho de pesquisa influencia e ajuda no progresso da sociedade, não só pela sua qualidade intrínseca, mas ao ser lido e citado em trabalhos subsequentes. Ou seja, os bons trabalhos tendem a tornar-se a semente para a continuação e aperfeiçoamento em pesquisas futuras. Na realidade, a norma do trabalho científico vigente assenta no uso e citação de conhecimento já existente. Ou seja, novas pesquisas assentam em pesquisas anteriores para a construção cumulativa e gradual de conhecimento. Assim, a dedicação aos elementos que fazem parte do contato inicial com o leitor e que podem impactar a sua decisão em dar continuidade à leitura dos artigos deve receber tanta atenção e rigor como os restantes elementos que compõem o corpo do artigo (revisão da literatura, hipóteses, método, resultados, discussão).

Nossa iniciativa em tratar destes aspectos nos comentários editoriais vem da nossa própria experiência como editores e como professores em programas de stricto sensu em Administração. Em especial como Professores notamos que tantas vezes é escasso o aprendizado de como redigir os artigos. Ou seja, os estudantes aprendem as teorias e os métodos, mas têm deficiências em trazer os seus conhecimentos para o artigo que acaba por ficar muito desconexo e com lacunas que podem levar à rejeição. Em especial, neste comentário quisemos focar estes três elementos título, resumo e palavras-chave - que são dos mais descurados, inclusive por pesquisadores experientes.

\section{REFERÊNCIAS}

Brito, R. \& Brito, R. (2012). Vantagem competitiva, criação de valor e seus efeitos sobre o desempenho. Revista de Administração de Empresas, 52 (1), 70-84.

Ferreira, M. (2013). O processo editorial: Da submissão à rejeição (ou aceite). Revista IberoAmericana de Estratégia, 12(3), 1-11.

Ferreira, M. \& Serra, F. (forthcoming) Estratégias de internacionalização sobre pressões institucionais: Um modelo conceitual sobre as imposições duais para legitimidade e conformidade. Revista de Administração Contemporânea.

Henisz, W., Dorobantu, S. \& Nartey, L. (2014). Spinning gold: The financial returns to stakeholder engagement. Strategic Management Journal, 35(12), 1727-1748

Hesson, J. (2013) English for research papers: a handbook for Brazilian authors. (disponível para Kindle na Amazon no link 
http://www.amazon.com.br/English-ResearchPapers-Handbook-Brazilianebook/dp/B00GPT0FXW/ref=sr_1_1?s=digital$\underline{\text { text\&ie }=U T F 8 \& q i d=1396928945 \& s r=1-}$ 1\&keywords=Research+papers).

King, A. \& Lepak, D. (2011). From the Editors: Debunking myths about AMR. Academy of Management Review, 36 (2), 207-214.

Kreitlon, M. (2012). McMoral, iPolítica, Cidadania Wireless: Reflexões para o ensino e a pesquisa em RSE no Brasil. Revista de Administração Contemporânea, 16(2), 271-289.

Lebrun, J-L. (2007). Scientific writing: A reader and writer's guide. Boston, MA: World Scientific.

Russell, A. (2004) Calibrated Peer ReviewTM: A writing and critical-thinking instructional tool. In Invention and impact: Building excellence in undergraduate science, technology, engineering and mathematics (STEM) education. Washington: American Association for the Advancement of Science.

Russey, W, Ebel, H. \& Bliefert, C. (2006). How to write a successful science thesis: The concise guide for students. New York, NY: Wiley-VCH.
Serra, F., Fiates, G. \& Ferreira, M. (2008). Publicar é difícil ou faltam competências? O desafio de pesquisar e publicar em revistas científicas na visão de editores e revisores internacionais. Revista de Administração McKenzie, 9(4), 32-55.

Serra, F., Ferreira, M. \& Almeida, M. (2013) Organizational decline: a yet largely neglected topic in organizational studies. Management Research: The Journal of the Iberoamerican Academy of Management, 11(2): 133-156

Shen, R., Tang, Y. \& Chen, G. (2014). When the role fits: How firm status differentials affect corporate takeovers. Strategic Management Journal, 35(13), 2012-2030.

Swales, J. \& Feaks, C. (2004) Academic writing for graduate students: essential skills and tasks. Ann Arbor: University of Michigan.

Teece, D., Pisano, G. \& Shuen, A. (1997) Dynamic capabilities and strategic management. Strategic Management Journal, 18(7): 509-533.

\footnotetext{
${ }^{\mathrm{i}}$ Lista de Comentários Editoriais já publicados:

Ferreira, M. (2013) A Pesquisa e a Estruturação do Artigo Acadêmico em Administração Revista Ibero-Americana de Estratégia, 12(2): 1-11.

Ferreira, M. (2013) O processo editorial: da submissão à rejeição (ou aceite). Revista Ibero-Americana de Estratégia, 12(3): 1-11.

Ferreira, M. (2013) A construção de hipóteses. Revista Ibero-Americana de Estratégia, 12(4): 1-8.

Ferreira, M. (2014) Responder aos revisores. Revista Ibero-Americana de Estratégia, 13(1): 1-6.

Ferreira, M. (2014) Como rever um artigo: O papel do revisor e um roteiro para novos revisores. Revista Ibero-Americana de Estratégia, 13(2): 1-9.

Ferreira, M. (2014) Resiliência e resistir à rejeição para o sucesso na carreira. Revista Ibero-Americana de Estratégia, 13(3): 1-6.

ii Este comentário editorial foi inspirado e teve como base para orientação o livro de Jim Hesson (2013), "English for research papers: A handbook for Brazilian authors" (disponível para Kindle na Amazon no link http://www.amazon.com.br/English-

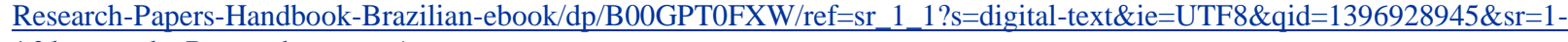

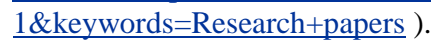

